\title{
Management of refractory lupus nephritis: challenges and solutions
}

This article was published in the following Dove Press journal:

Open Access Rheumatology: Research and Reviews

JH Yo

TD Barbour

$K$ Nicholls

Department of Nephrology, The Royal Melbourne Hospital, Melbourne, Victoria, Australia
Correspondence: K Nicholls Royal Melbourne Hospital, 300 Grattan Street, Parkville, VIC 3050, Australia Tel +6I 0393427000

Email Kathy.nicholls@mh.org.au

\begin{abstract}
Refractory lupus nephritis, broadly defined as failure to attain clinical remission after appropriate induction immunosuppressive therapy, is associated with an increased risk of progression to end-stage kidney disease and mortality. This is a challenging issue in clinical practice, as modern induction therapy despite proven efficacy can still be associated with treatment failure. Moreover, newer therapies have failed in recent years to displace or even match existing protocols for effective induction of remission. Refractory disease is generally assessed on the basis of clinical parameters, which may be unreliable, and renal biopsy, which is often not performed in a standard or timely fashion. Persisting histological inflammation in $30 \%-50 \%$ of patients who have attained clinical remission highlights the disparity between clinical and immunological response to therapy. The lack of an international consensus regarding what constitutes refractory lupus nephritis compounds clinician indecision regarding optimal management for these patients. Moreover, non-adherence to prescribed therapy versus primary treatment failure can be challenging to discriminate, and the time point at which non-response becomes treatment failure is unclear. In this review, we assess the key published evidence for the treatment of refractory lupus nephritis and provide practical recommendations based around the use of adjunctive therapies. These agents include rituximab and calcineurin inhibitors, with evidence consisting largely of observational or uncontrolled studies, as well as some of the biologic therapies currently under investigation through prospective clinical trials. The poor prognosis of refractory lupus nephritis demands regular review of patient response and the flexibility to switch or augment therapy.
\end{abstract}

Keywords: lupus nephritis, refractory, management

\section{Introduction}

Lupus nephritis (LN) is a common severe complication of systemic lupus erythematosus (SLE) and a major determinant of morbidity and mortality. ${ }^{1}$ Up to $60 \%$ of adult patients with $\mathrm{SLE}^{2,3}$ develop renal disease of differing severity during the course of their illness. Despite the introduction of corticosteroids and other immunosuppressive agents which have profoundly changed the management of LN, namely cyclophosphamide (CYP) and mycophenolate mofetil (MMF), between $20 \%$ and $70 \%$ of patients with LN are reported to be refractory to standard immunosuppressive therapy. ${ }^{4}$ The variable response rates reported in the literature are due to several factors including length of follow up, different definitions of clinical response used, and the ethnicity of the cohort being studied.

Patients with refractory LN have poorer outcomes. For example, in a study of 86 patients with diffuse proliferative LN, the patient survival at 10 years was $95 \%$ for 
complete remission, $76 \%$ for partial remission and $46 \%$ for no remission. Renal survival at 10 years was $94 \%$ for complete remission, $45 \%$ for partial remission and $19 \%$ for no remission. ${ }^{5,6}$ Thus, even a partial remission in $\mathrm{LN}$ is associated with significantly better outcomes compared with no remission.

Long-term high dose immunosuppression is also associated with significant treatment toxicity. Novel alternative therapies that are currently available include "multitarget therapy" and various biologics that target B cells, T cells or cytokines, but to date, no new approaches to the treatment of LN have been shown to be superior to CYC or MMF plus corticosteroids.

The goals of this review are to address the key issues in the assessment and management of refractory LN, including the definition of complete response, the role of repeat renal biopsy to inform clinical decision-making, and the evidence for adjunctive therapies for patients with refractory disease.

\section{Definition of refractory LN}

A major challenge in determining the optimal treatment approach for refractory $\mathrm{LN}$ is the lack of a consensus definition for complete response following induction treatment. As a result, the reported incidence of "refractory" $\mathrm{LN}$ varies according to which of several definitions for complete remission is used. The European League Against Rheumatism (EULAR/ERA-EDTA) recommendations consider refractory $\mathrm{LN}$ as those patients who do not achieve a partial response after 6-12 months. ${ }^{7}$ The guidelines from the American College of Rheumatology have defined refractory LN as worsening nephritis by 3 months or, treatment failure as assessed by the treatment physician by 6 months. ${ }^{8}$

Most criteria for complete or partial response are a composite of clinical indices including serum creatinine, proteinuria and urinary red blood cells. The Kidney Disease and Improving Global Outcomes (KDIGO) guideline on glomerulonephritis defines complete remission as return of serum creatinine to previous baseline and a decline in the urine protein creatinine ratio (UPCR) to $<500 \mathrm{mg} / \mathrm{mmol}$. Partial remission is defined by stabilization $( \pm 25 \%)$ or improvement of serum creatinine, but not to normal, and a $>50 \%$ decrease in the UPCR. ${ }^{9}$ In clinical practice, partial remission is expected at 3-6 months, and clinical parameters are usually assessed every 4 weeks in the first 6 months.

There are several problems with the focus on decline in proteinuria and creatinine as a marker of remission. Firstly, emerging data on protocol repeat biopsies performed after complete clinical remission show continuing histologic activity in a significant number of patients. ${ }^{10-13}$ Despite the discordance between histological and clinical metrics of disease activity, renal response definitions do not routinely include renal histology as a component of assessment. Stopping maintenance therapy based on clinical activity indices alone may theoretically put such patients at risk of a renal flare.

Secondly, there are other non-immunologic mechanisms of renal injury which may lead to changes in creatinine and proteinuria including thrombotic microangiopathy and secondary focal glomerulosclerosis. Moreover, all calcineurin inhibitors (CNI) therapy, potential therapy for LN, act directly on podocytes to reduce proteinuria, independent of its immunosuppressive effects. Thus, it is unclear whether disease remissions observed with CNI therapy result from "true" remission of the immunological disease processes or are the result of the antiproteinuric effects of CNIs.

Thirdly, the clinical intersection between what is sometimes termed "true" refractory $\mathrm{LN}$, as opposed to nonadherence to therapy or inadequacy of the prescribed regimen, is somewhat blurred. Several studies suggest that problems with treatment adherence may directly influence clinical outcomes in patients with SLE. ${ }^{14,15}$ Important barriers to adherence have been identified in focus groups and include financial problems, fear of side effects, difficulties navigating the health system and perceived treatment inefficacy. ${ }^{16}$

In practical terms, it can be extremely difficult to know whether a patient is non-adherent to treatment. This has significant implications for the intensification of therapy and accompanying drug toxicities, switching of therapy, and the monitoring of such patients. Pill counts, pharmacy records and measuring therapeutic levels of drugs (eg, MMF) may assist in the assessment of adherence but is no guarantee that the prescribed therapy has been taken. One exception may be in those patients receiving intravenous CYC, which is sometimes preferred as induction specifically because adherence is intrinsic.

Conversely, in an adherent patient failing to achieve remission, insufficient dosing of first line induction therapies should be considered. It is therefore important to review dosages of prescribed agents to ensure they conform with evidence-based guideline recommendations. ${ }^{8,9,17}$ For example, if a patient is on MMF for induction of $2 \mathrm{~g}$ /day and has not achieved remission, we would recommend maximizing the dose to $3 \mathrm{~g} /$ day, as tolerated. 
Nonetheless, therapeutic drug monitoring by assay of the 12 hour MMF area under the curve for dosage titration has shown some promise in $\mathrm{LN}^{18}$ A tailored approach such as this may be of use, for example, in Asian patients who are more susceptible to adverse effects of MMF at higher dosages prescribed on a per protocol basis.

In this review, we use the term refractory $\mathrm{LN}$ in reference to adherent patients who fail to achieve either a complete or partial response to initial standard immunosuppressive therapy, or whose renal function deteriorates on therapy, within a 6 to 12-month period. Long-term follow-up of patients in the Euro Lupus Nephritis Trial showed that the best predictor of good long-term renal outcomes is early (3-6 months) reduction in proteinuria by at least $50 \%$ or to $<1 \mathrm{~g} / 24$ hours and decrease in creatinine, ${ }^{19}$ a finding validated in subsequent studies. ${ }^{20}$ Thus, clinical response to primary induction therapy after a 6-month interval represents an important checkpoint for the treating physician.

\section{The role of the kidney biopsy in refractory LN}

There are a number of key clinical circumstances, after initial histological diagnosis and staging of $\mathrm{LN}$, at which repeat renal biopsy may provide useful information when considering a diagnosis of refractory LN.

A renal biopsy should be considered for patients on induction therapy presenting with worsening proteinuria and creatinine. Whilst immune complex-mediated LN is the most common cause of kidney disease in SLE, other mechanisms can result in renal injury that are not apparent by clinical data alone and require a different management approach. Examples include thrombotic microangiopathy, lupus podocytopathy, antiphospholipid antibody-induced vascular lesions, and tubulointerstitial nephritis.

For patients with worsening clinical parameters, refractory LN must be considered. We recommend repeat renal biopsy for patients who have not met the clinical criteria for complete or partial remission to first line induction immunosuppression within 6-12 months. Deciding when to repeat a biopsy for suspicion of refractory disease is challenging, and ultimately must be individualized to the patient and overall clinical assessment. For example, some clinicians may elect to wait 12 months or longer if creatinine is stable and proteinuria is progressively declining. Conversely, rapid decline of clinical parameters may necessitate an early repeat biopsy even before the 6 to 12 - month period. Our own practice is to consider repeat biopsy at 6 months if there has been no clear improvement, and earlier if renal function worsens on standard therapy over the first 3 months.

For patients with slowly rising serum creatinine or persistent proteinuria, a repeat renal biopsy is also helpful to distinguish active proliferative $\mathrm{LN}$ from advanced sclerosing LN which may allow for reduction in immunosuppression. Studies have demonstrated that increasing chronic damage on repeat biopsies performed after at least 6 months of immunosuppressive therapy is predictive of long-term renal outcomes. ${ }^{10,12}$

The role of protocol repeat kidney biopsies in LN is still debated and local preferences predominate. Emerging data suggests that serial biopsies may inform ongoing treatment decisions. After completing 6-8 months of immunosuppressive therapy, $30-50 \%$ of complete clinical renal responders still had histologic evidence of ongoing active inflammation, and $40-60 \%$ of patients with no histologic evidence of disease activity still had persistent high-grade proteinuria. ${ }^{10,11}$ Despite several years of immunosuppressive therapy and at least 24 months of clinically quiescent disease, histologic activity was found in $30 \%$ of $\mathrm{LN}$ patients. ${ }^{11}$

A more comprehensive picture of kidney pathology has been examined through molecular imaging of the kidney biopsy. A recent study of intrarenal transcript expression profiles of the diagnostic kidney biopsy in patients with LN showed differing molecular profiles between patients who had a rapid clinical response to induction therapy and patients who did not. ${ }^{21}$ The addition of molecular evaluation to histology has the potential to facilitate the individualization of LN treatment and identify those patients who would be refractory to conventional therapy.

\section{The implications of refractory LN}

The importance of achieving a complete or partial response was illustrated by the Lupus Nephritis Collaborative Study Group. Patients who achieved complete remission had better 10-year patient and renal survival rates (95\% and $94 \%$, respectively) as compared to those who attained partial remission $(76 \%$ and $45 \%$, respectively). ${ }^{6}$ For patients who had no response to therapy, the 10-year patient and survival rate was $46 \%$ and $13 \%$ respectively. ${ }^{5}$ Thus, even a partial response is better than no response in patients with proliferative LN.

Prognosis in LN is also influenced by the heterogenous demographic, clinical and histological variables of this disease. African American and Hispanic ethnicity were associated with 
a shorter time-to-LN occurrence $(\mathrm{HR}=2.57,95 \%$ CI 1.26-5.26; $p=0.001$ and HR 2.32, 95\% CI 1.03-5.25; $p=0.001$ respectively) compared with Caucasians. ${ }^{22}$ Indeed, African Americans with LN had the worst outcomes when compared to Hispanics and Caucasians, with the development of renal events or death being three times higher in African Americans and two times higher in Hispanics when compared to Caucasians. ${ }^{23}$ Dooley $^{24}$ also reported a significantly poorer renal survival (free of ESRD) in African Americans compared to Caucasians treated with cyclophosphamide for LN, with a 5-year renal survival of 58\% in African Americans as compared to $95 \%$ in Caucasians. ${ }^{24}$ The reasons for these disparate outcomes remain controversial.

A higher incidence and more severe nephritis are also seen in male gender and juvenile onset SLE. ${ }^{3}$ Other clinical factors include elevated serum creatinine at time of presentation, hypertension and tubulointerstitial inflammation. $^{23,25,26}$ Australian data on LN is scarce, with some studies suggesting that Asian Australians were more affected by SLE than non-Asian Australians in terms of disease severity and renal involvement. ${ }^{27,28}$

\section{Therapeutic options for refractory LN}

The management of patients with refractory disease varies with the first line agent used for induction therapy, clinical factors and local practices as outlined below. A suggested approach to the assessment and management of refractory LN is detailed in Figure 1.

\section{Switching from CYC to MMF or vice versa}

Switching to another first line induction agent is the recommended initial approach for patients with refractory LN and is recommended by both the EULAR/ERA-EDTA and American College of Rheumatology guidelines. ${ }^{1,17}$ In general, CYC-resistant patients are treated with MMF and MMF-resistant patients with CYC. In addition to switching immunosuppressive agents, some accompany this with 3 days of intravenous pulses of glucocorticoids. ${ }^{8}$

The efficacy of MMF in patients with refractory $\mathrm{LN}^{29-33}$ has been demonstrated in several open label studies. Rivera et $\mathrm{al}^{34}$ examined 85 patients with relapsing $(n=50)$ and refractory $(n=35$, who had not responded after 6 months of therapy) LN who were switched to MMF induction, of whom $87 \%$ had received initial induction therapy with IV CYC. Overall, 74 patients $(87 \%)$ achieved a response $(69 \%$ partial and $31 \%$ complete). ${ }^{34}$

Dooley et $\mathrm{al}^{30}$ reported improvements in renal function and proteinuria in 12 patients given MMF who were either

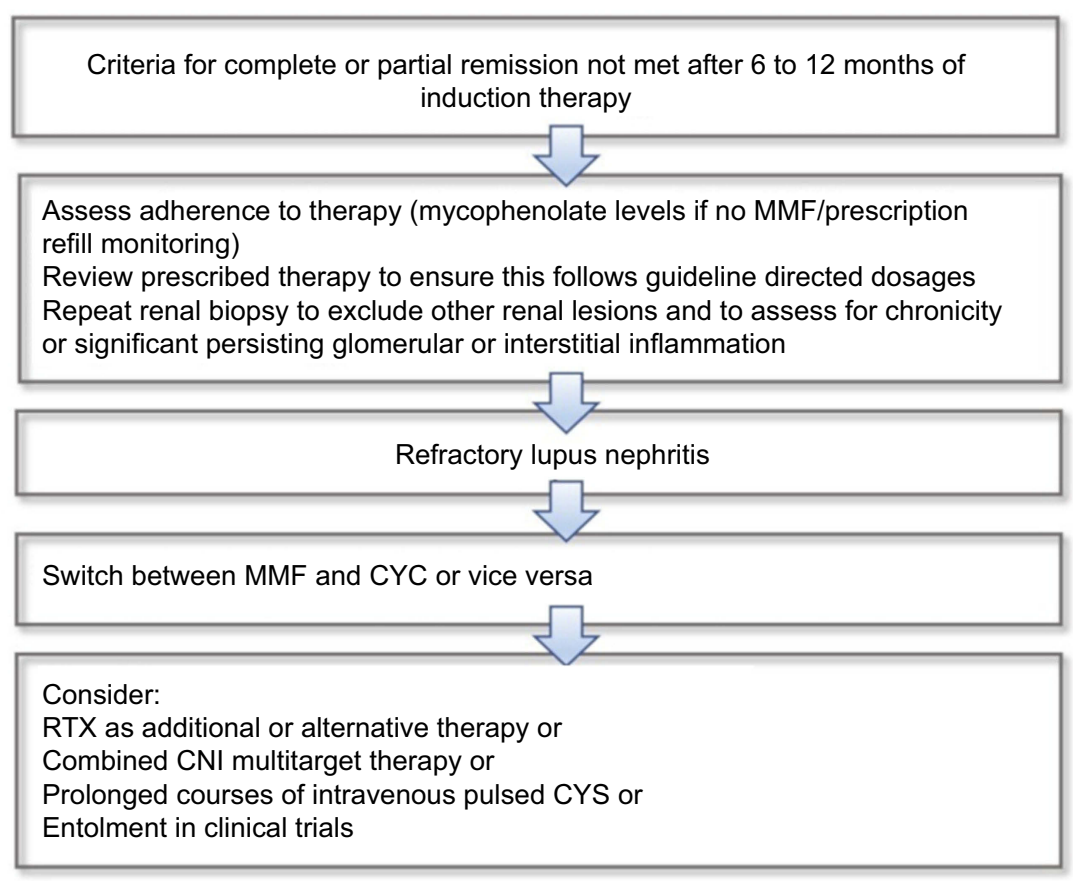

Figure I A suggested treatment algorithm for refractory lupus nephritis.

Abbreviations: MMF, mycophenolate; CYC, cyclophosphamide; CNI, calcineurin inhibitors; RTX, rituximab. 
refractory or relapsed after treatment with $\mathrm{CYC} .{ }^{30}$ Four of the patients had declining renal function while receiving initial therapy with CYP and two patients had relapsing LN.

The opposite approach (switching from MMF to CYP) is also recommended ${ }^{8,9,17}$ based on good long-term efficacy data of CYC in LN despite there being no data on outcomes of patients who have made this switch. Additionally, for a patient with suspected nonadherence to an induction regimen consisting of MMF or oral cyclophosphamide, switching to IV cyclophosphamide may be helpful.

\section{Prolonged courses of cyclophosphamide}

Extended therapy with CYC can be considered for patients who fail to meet remission criteria by 6 months. Mok et $\mathrm{al}^{35}$ demonstrated in a cohort of Chinese patients with LN that cumulative doses, determined the initial treatment response. ${ }^{35}$ However, the significant treatment toxicities of CYC, such as infertility, urotoxicity and oncogenicity, which are particularly relevant as most patients are of childbearing age, is a major limitation to this. Ovarian reserve is inversely related to the cumulative doses of $\mathrm{CYC}$ received and concomitant use of gonadotropin-releasing hormone $(\mathrm{GnRH})$ analogues should be considered. ${ }^{36,37}$

\section{B cell therapies}

B cells play a principal role in the pathogenesis of $\mathrm{LN}$ and are therefore attractive therapeutic targets. Rituximab (RTX) is a chimeric mouse-human monoclonal antibody directed against the B-cell surface molecule CD20. Several uncontrolled studies reported efficacy of RTX in patients with refractory LN. ${ }^{38-45}$ However, RTX failed to show any benefit in the Exploratory Phase II/III SLE Evaluation of Rituximab (EXPLORER) trial which excluded patients with severe active LN. ${ }^{46}$ Additionally, the single randomized, double-blind placebo-controlled trial (LUNAR) comparing RTX with placebo when added to standard of care with prednisolone and MMF in incident LN, also failed to demonstrate any significant therapeutic efficacy. ${ }^{47}$

Interpretation of these results needs to be balanced with the concerns raised about the LUNAR trial's design. All patients received intense background immunosuppression with regular infusions of $1 \mathrm{~g}$ methylprednisolone and high dose oral MMF which may explain the minimal additional benefit seen with the addition of RTX at week 52 follow up. In addition, the LUNAR trial included only incident LN patients, whereas most previous uncontrolled studies describe efficacy in a cohort of patients with refractory LN who had failed CYC or MMF and had RTX as an alternative, not an add-on therapy. ${ }^{48}$

Additionally, a higher complete response rate was seen with respect to proteinuria in patients who received RTX (32 vs 9\%). This reduction in proteinuria persisted through to 78 weeks, raising the possibility that a longer period of follow up may have yielded a significant difference. The study also found higher partial response rates in the RTX group for all patients and in the prespecified subgroup of African-American patients, however, the trial was not powered to detect differences in partial response rates. ${ }^{47}$

Gomez-Mendez et $\mathrm{al}^{49}$ recently demonstrated in a post hoc analysis of the LUNAR trial that there was substantial variability in peripheral blood B cell depletion in patients with LN treated with RTX. Achievement of complete peripheral depletion (peripheral $B$ cells 0 cells $/ \mu \mathrm{L}$ ) as well as the rapidity and duration of complete peripheral depletion were associated with complete response at week 78. ${ }^{49}$ Reasons for this have been hypothesized to include autoreactive pathogenic B cells persisting within lymphoid structures and the kidney tubulointerstitium. ${ }^{50,51}$

Despite the failure of the LUNAR trial to meet its primary endpoint, advocacy for the use of RTX in refractory LN continues and is primarily based on observational evidence, especially in patients who have an inadequate response to first line induction regimens. In a systematic review of published case reports and case series on the efficacy of RTX in patients with refractory LN, 300 patients who were followed up for 60 weeks had sustained complete and partial response rates in 87, 76, 67 and 76\% of patients with class III, IV, $\mathrm{V}$ and mixed class respectively. $^{48}$

A few cases of progressive multifocal leukoencephalopathy caused by reactivation of polyoma virus have been reported in patients with rheumatologic disease treated with RTX. ${ }^{52}$ Many of these patients were also on other immunosuppressive agents, and it is possible that reactivation risk is determined by total immunosuppression rather than by any specific drug alone.

The exact role of RTX in the treatment paradigm of LN remains to be determined with open label evidence suggestive of a role in the treatment of refractory LN. RTX is a reasonable alternative therapy that could be considered for patients with refractory disease.

\section{Calcineurin inhibitors}

The calcineurin inhibitors (CNIs) cyclosporine A (CSA) and tacrolimus have been studied extensively in LN, 
especially in Asia, with emerging evidence for its efficacy in refractory $\mathrm{LN}$.

CSA binds to its cytoplasmic receptor, cyclophyllin, and the resultant drug-receptor complex binds to calcineurin and interferes with IL-2 production, thus leading to selective and reversible inhibition of $\mathrm{T}$ cell-mediated immune response. In addition to its potent immunosuppressive action, CSA has been shown to have direct antiproteinuric effects. The mechanism is related to stabilization of actin in podocytes. ${ }^{53}$ Tacrolimus binds to a different cytoplasmic receptor, the FK-binding protein12 , which then interacts with calcineurin.

The CNIs have shown encouraging results in open label trials and are a potential less toxic alternative to conventional therapy. An early trial of 18 patients with proliferative $\mathrm{LN}$ refractory to conventional therapy who were administered CSA at $5 \mathrm{mg} / \mathrm{kg} /$ day had reduced proteinuria, improved renal function and decreased requirement for corticosteroids. ${ }^{54}$ Dostal et $\mathrm{al}^{55}$ demonstrated the efficacy of CSA administered for 1 year to eleven patients with LN (eight with LN refractory or intolerant to CYC or azathioprine). ${ }^{55}$ There was a significant improvement in disease activity scores, degree of proteinuria, dsDNA titres and histological activity. In a small open label prospective study, nine Japanese patients with LN refractory to conventional therapy received low dose CSA $(2.5 \mathrm{mg} / \mathrm{kg} /$ day $)$ and were followed up for 30 weeks. Two patients achieved complete remission and four went into partial remission with improved proteinuria and reduced corticosteroid dependence. $^{56}$

CNIs have been used as part of a multitarget approach to treat $\mathrm{LN}$, added to a regimen of MMF and corticosteroids. Combining immunosuppressive agents for a synergistic effect has long been utilized in organ transplantation to minimize the risk of rejection. Multitarget therapy comprising corticosteroids, tacrolimus and MMF has been investigated in a prospective randomized open label trial for the induction treatment of $\mathrm{LN}$ and has been shown to be superior to intravenous CYC in induction of remission at 6 months. ${ }^{57}$ Interestingly, there were more patient withdrawals due to adverse events in the CNI arm than the CYC arm, though the overall rate of adverse events did not differ between the two groups. These results need to be interpreted cautiously as reduction in proteinuria is the major clinical determinant of renal remission in these trials and CNIs can attenuate proteinuria through immune and nonimmune mechanisms. Indeed, upon cessation of these agents, the antiproteinuric affects are often reversed. Thus, proteinuria may not be an appropriate end point in LN patients treated with a CNI, and post-treatment kidney biopsy should be performed to ensure resolution of immune injury. Finally, long-term preservation of kidney function and applicability of CNIs to non-Asian cohorts need to be verified.

\section{Plasma exchange and immunoadsorption}

The evidence for extracorporeal treatments such as plasma exchange and immunoadsorption in refractory LN is minimal, based mainly on single case reports and observational studies. A randomized, controlled trial conducted by the Lupus Nephritis Collaborative Study Group examining the efficacy of plasma exchange when added to standard therapy in patients with severe LN failed to show improvement in clinical outcomes. ${ }^{58}$ In a study of eight patients with refractory disease who underwent immunoadsorption and had concomitant CYC, all patients had reductions in creatinine and urinary protein excretion. ${ }^{59}$

Several studies have reported that plasma exchange in antiphospholipid syndrome and its associated complications may be of benefit. ${ }^{60,61}$ Extracorporeal therapies have sometimes been considered for patients with contraindications to standard therapy including severe infection risk and significant leucopenia as well as other indications such as pulmonary hemorrhage. ${ }^{60}$

\section{Investigational biologic therapies}

A number of clinical trials on the efficacy and safety of emerging novel biologic therapies are under way. Access to such agents are limited to trials at this stage and consideration of trial therapy may be a therapeutic option for those patients with refractory LN who have failed to respond to or are intolerant of the above regimens. It should be noted that the efficacy of these novel biologic agents has not been studied in a cohort of patients with refractory disease.

\section{B cell survival factors}

B lymphocyte stimulator (BLyS) and a proliferationinducing ligand (APRIL) are key survival cytokines for B cell survival and development. They bind to three different receptors: transmembrane activator and calcium-modulating cytophilin ligand interactor (TACI), the B cell maturation antigen (BCMA) and the BAFF receptor (BAFF-R). ${ }^{62}$

Overexpression of BLyS promotes survival of $B$ cells (including autoreactive B cells), whereas inhibition of BLyS results in autoreactive B cell apoptosis. Elevated circulating BLyS and APRIL levels are seen in SLE and 
correlate with increased SLE disease activity and elevated anti-double stranded DNA (anti-dsDNA) antibody concentrations. $^{63,64}$

Belimumab is the first new drug approved by both the Food and Drug Administration (FDA) and by the European Medicines Agency for antibody-positive SLE in decades. Belimumab is a human monoclonal IgG1 antibody that binds to and inhibits soluble B-lymphocyte stimulator protein. It was found to be superior compared with placebo in two Phase III placebo-controlled trials, BLISS-52 and BLISS-76 in addition to standard therapy in 1684 serologically positive SLE patients. ${ }^{65,66}$

Although patients with severe LN were excluded from this study, a pooled post-hoc analysis of the cohort indicated that over 52 weeks there was a significant reduction in the frequency of renal flares and a tendency for greater reduction in proteinuria. ${ }^{67} \mathrm{~A}$ phase 3 randomized controlled trial is currently underway to evaluate the efficacy of belimumab in LN (NCT01639339).

\section{Co-stimulation blockade}

Abatacept is a soluble Fc CTLA-4 fusion protein that prevents $\mathrm{T}$ cell activation by competing with CD28 for binding to CD80-86, a costimulatory signal required for T cell activation.

Evidence from the Abatacept and Cyclophosphamide Combination Therapy for Lupus Nephritis (ACCESS) trial, failed to demonstrate any benefit of abatacept plus low dose CYC compared to placebo plus low dose CYC. ${ }^{68}$ Interestingly, patients on the abatacept arm who reached complete renal remission at 6 months were followed for another 6 months without any maintenance immunosuppressive therapy. At 12 months, the patients in the abatacept arm had fewer renal flares. Whilst the study was not powered to test abatacept for maintenance of remission, these results suggest abatacept may be worth investigating as a maintenance agent for flare minimization.

Recently completed, the largest randomized controlled trial in LN (NCT01714817) had 405 participants and sought to compare abatacept to placebo when added to standard of care therapy consisting of mycophenolate and steroids. Disappointingly, there was no difference in the primary end point of complete renal remission at week 52 (abatacept $35 \%$ and placebo $33 \%, p=0.73$ ). ${ }^{69}$

\section{Epratuzumab}

Epratuzumab is a humanized monoclonal antibody of the IgG1 class that targets CD22 on B cells, disrupting the B cell receptor signaling complex and resulting in modulation of
$B$ cell activity without substantial reductions in the number of peripheral B cells. ${ }^{70}$ The EMBODY 1 and EMBODY 2 phase III multicenter randomized placebo-controlled doubleblinded trial was conducted in patients with moderately to severely active SLE, though excluded patients with $\mathrm{LN}^{71}$ Treatment with epratuzumab in conjunction with standard therapy did not result in improved outcomes at week 48 when compared to placebo. Phase III trials in LN have yet to be conducted.

\section{Atacicept}

Atacicept is a recombinant fusion protein comprising the extracellular domain of the TACI receptor joined to a human IgG1 Fc domain. ${ }^{62}$ Atacicept blocks B cell stimulation by both BLyS and APRIL pathways. In 306 patients with active, autoantibody positive non-renal SLE receiving standard of care, there was a trend towards efficacy when compared to placebo. ${ }^{72}$

\section{Anifrolumab}

Interferon-alpha appears to have a central regulatory role in SLE and LN and is therefore a potential treatment target. Recent phase III clinical trial, the TULIP-1 study, examined the efficacy of anifrolumab, a monoclonal antibody against IFN-alpha receptor 1, versus placebo in patients with moderately-severely active auto-antibodypositive non-LN SLE and, failed to meet the primary endpoint of a reduction of disease activity as measured by the SLE Responder Index (NCT02446899). The TULIP-2 trial and TULIP-LN1 trial, examining the efficacy of anifrolumab in active proliferative $\mathrm{LN}$, is ongoing (NCT02446912, NCT02547922).

In summary, LN that is refractory to first line induction therapies is associated with worse long-term renal and patient outcomes. The traditional clinical parameters used to assess clinical response cannot reliably distinguish immunological activity from persistent damage and scarring. A number of therapeutic strategies may be tried for patients with refractory disease, including using alternative first line induction agents, rituximab, calcineurin inhibitors or enrolment in clinical trials of new targeted therapeutics. Further evidence is needed to advance this topic forward from opinion-based to evidence-based recommendations. Only then can the significant clinician uncertainty that dominates the management of refractory LN be adequately addressed.

\section{Disclosure}

The authors report no conflicts of interest in this work. 


\section{References}

1. Bernatsky S, Boivin JF, Joseph L, et al. Mortality in systemic lupus erythematosus. Arthritis Rheum. 2006;54(8):2550-2557. doi:10.1002/ art. 21955

2. Borchers AT, Keen CL, Shoenfeld Y, Gershwin ME. Surviving the butterfly and the wolf: mortality trends in systemic lupus erythematosus. Autoimmun Rev. 2004;3(6):423-453. doi:10.1016/j.autrev.2004.04.002

3. Brunner HI, Gladman DD, Ibanez D, Urowitz MD, Silverman ED. Difference in disease features between childhood-onset and adult-onset systemic lupus erythematosus. Arthritis Rheum. 2008;58 (2):556-562. doi:10.1002/art.23204

4. Ginzler EM, Dooley MA, Aranow C, et al. Mycophenolate mofetil or intravenous cyclophosphamide for lupus nephritis. $N$ Engl J Med. 2005;353(21):2219-2228. doi:10.1056/NEJMoa043731

5. Chen YE, Korbet SM, Katz RS, Schwartz MM, Lewis EJ. Value of a complete or partial remission in severe lupus nephritis. Clin $\mathrm{J} \mathrm{Am}$ Soc Nephrol. 2008;3(1):46-53. doi:10.2215/CJN.03280807

6. Korbet SM, Lewis EJ, Schwartz MM, Reichlin M, Evans J, Rohde RD. Factors predictive of outcome in severe lupus nephritis. Lupus Nephritis Collaborative Study Group. Am J Kidney Dis. 2000;35(5):904-914.

7. Gordon C, Jayne D, Pusey C, et al. European consensus statement on the terminology used in the management of lupus glomerulonephritis. Lupus. 2009;18(3):257-263. doi:10.1177/0961203308100481

8. Hahn BH, McMahon MA, Wilkinson A, et al. American College of Rheumatology guidelines for screening, treatment, and management of lupus nephritis. Arthritis Care Res (Hoboken). 2012;64 (6):797-808. doi:10.1002/acr.21664

9. Kidney Disease: Improving Global Outcomes Group. KDIGO clinical practice guideline for glomerulonephritis. Kidney Int Suppl. 2012;2(2):139-274.

10. Zickert A, Sundelin B, Svenungsson E, Gunnarsson I. Role of early repeated renal biopsies in lupus nephritis. Lupus Sci Med. 2014;1(1): e000018. doi:10.1136/lupus-2014-000018

11. Alvarado AS, Malvar A, Lococo B, et al. The value of repeat kidney biopsy in quiescent Argentinian lupus nephritis patients. Lupus. 2014;23(8):840-847. doi:10.1177/0961203313518625

12. Malvar A, Pirruccio P, Alberton V, et al. Histologic versus clinical remission in proliferative lupus nephritis. Nephrol Dial Transplant. 2017;32(8):1338-1344. doi:10.1093/ndt/gfv296

13. Alsuwaida A, Husain S, Alghonaim M, et al. Strategy for second kidney biopsy in patients with lupus nephritis. Nephrol Dial Transplant. 2012;27(4):1472-1478. doi:10.1093/ndt/gfr517

14. Petri M, Perez-Gutthann S, Longenecker JC, Hochberg M. Morbidity of systemic lupus erythematosus: role of race and socioeconomic status. Am J Med. 1991;91(4):345-353.

15. Adler M, Chambers S, Edwards C, Neild G, Isenberg D. An assessment of renal failure in an SLE cohort with special reference to ethnicity, over a 25-year period. Rheumatology (Oxford). 2006;45 (9):1144-1147. doi:10.1093/rheumatology/kel039

16. Garcia Popa-Lisseanu MG, Greisinger A, Richardson $M$, et al. Determinants of treatment adherence in ethnically diverse, economically disadvantaged patients with rheumatic disease. $J$ Rheumatol. 2005;32(5):913-919.

17. Bertsias GK, Tektonidou M, Amoura Z, et al. Joint European League against Rheumatism and European Renal Association-European Dialysis and Transplant Association (EULAR/ERA-EDTA) recommendations for the management of adult and paediatric lupus nephritis. Ann Rheum Dis. 2012;71(11):1771-1782. doi:10.1136/annrheumdis-2012-201940

18. Zahr N, Arnaud L, Marquet P, et al. Mycophenolic acid area under the curve correlates with disease activity in lupus patients treated with mycophenolate mofetil. Arthritis Rheum. 2010;62 (7):2047-2054. doi:10.1002/art.27495
19. Houssiau FA, Vasconcelos C, D'Cruz D, et al. Early response to immunosuppressive therapy predicts good renal outcome in lupus nephritis: lessons from long-term followup of patients in the Euro-Lupus nephritis trial. Arthritis Rheum. 2004;50 (12):3934-3940. doi:10.1002/art.20666

20. Korbet SM, Lewis EJ. Severe lupus nephritis: the predictive value of a $>/=50 \%$ reduction in proteinuria at 6 months. Nephrol Dial Transplant. 2013;28(9):2313-2318. doi:10.1093/ndt/gft201

21. Parikh SV, Malvar A, Song H, et al. Molecular imaging of the kidney in lupus nephritis to characterize response to treatment. Transl Res. 2017;182:1-13. doi:10.1016/j.trs1.2016.10.010

22. Burgos PI, McGwin G Jr., Pons-Estel GJ, Reveille JD, Alarcon GS, Vila LM. US patients of Hispanic and African ancestry develop lupus nephritis early in the disease course: data from LUMINA, a multiethnic US cohort (LUMINA LXXIV). Ann Rheum Dis. 2011;70(2):393-394. doi:10.1136/ard.2010.131482

23. Contreras G, Lenz O, Pardo V, et al. Outcomes in African Americans and Hispanics with lupus nephritis. Kidney Int. 2006;69 (10):1846-1851. doi:10.1038/sj.ki.5000243

24. Dooley MA, Hogan S, Jennette C, Falk R. Cyclophosphamide therapy for lupus nephritis: poor renal survival in black Americans. Glomerular disease collaborative network. Kidney Int. 1997;51 (4):1188-1195.

25. Austin HA 3rd, Klippel JH, Balow JE, et al. Therapy of lupus nephritis. Controlled trial of prednisone and cytotoxic drugs. $N$ Engl J Med. 1986;314(10):614-619. doi:10.1056/NEJM1986 03063141004

26. Hsieh C, Chang A, Brandt D, Guttikonda R, Utset TO, Clark MR. Predicting outcomes of lupus nephritis with tubulointerstitial inflammation and scarring. Arthritis Care Res (Hoboken). 2011;63 (6):865-874. doi:10.1002/acr.20441

27. Golder V, Connelly K, Staples M, Morand E, Hoi A. Association of Asian ethnicity with disease activity in SLE: an observational study from the Monash Lupus Clinic. Lupus. 2013;22(13):1425-1430. doi:10.1177/0961203313500547

28. Ong C, Nicholls K, Becker G. Ethnicity and lupus nephritis: an Australian single centre study. Intern Med J. 2011;41(3):270-278. doi:10.1111/j.1445-5994.2009.02159.x

29. Ding L, Zhao M, Zou W, Liu Y, Wang H. Mycophenolate mofetil combined with prednisone for diffuse proliferative lupus nephritis: a histopathological study. Lupus. 2004;13(2):113-118. doi:10.1191/ 0961203304lu513oa

30. Dooley MA, Cosio FG, Nachman PH, et al. Mycophenolate mofetil therapy in lupus nephritis: clinical observations. J Am Soc Nephrol. 1999;10(4):833-839.

31. Kapitsinou PP, Boletis JN, Skopouli FN, Boki KA, Moutsopoulos HM. Lupus nephritis: treatment with mycophenolate mofetil. Rheumatology (Oxford). 2004;43(3):377-380. doi:10.1093/ rheumatology/keh012

32. Kingdon EJ, McLean AG, Psimenou E, et al. The safety and efficacy of MMF in lupus nephritis: a pilot study. Lupus. 2001;10(9):606-611. doi:10.1191/096120301682430186

33. Li L, Wang H, Lin S, et al. Mycophenolate mofetil treatment for diffuse proliferative lupus nephritis: a multicenter clinical trial in China. Zhonghua Nei Ke Za Zhi. 2002;41(7):476-479.

34. Rivera F, Merida E, Illescas ML, et al. Mycophenolate in refractory and relapsing lupus nephritis. Am J Nephrol. 2014;40(2):105-112. doi:10.1159/000365256

35. Mok CC, Ying KY, Ng WL, et al. Long-term outcome of diffuse proliferative lupus glomerulonephritis treated with cyclophosphamide. Am $J$ Med. 2006;119(4):355.e25-e33. doi:10.1016/j.amjmed.2005.08.045

36. Mok CC, Chan PT, To CH. Anti-mullerian hormone and ovarian reserve in systemic lupus erythematosus. Arthritis Rheum. 2013;65 (1):206-210. doi:10.1002/art.37719 
37. Blumenfeld Z, Shapiro D, Shteinberg M, Avivi I, Nahir M. Preservation of fertility and ovarian function and minimizing gonadotoxicity in young women with systemic lupus erythematosus treated by chemotherapy. Lupus. 2000;9(6):401-405. doi:10.1191/ 096120300678828596

38. Gunnarsson I, Sundelin B, Jonsdottir T, Jacobson SH, Henriksson EW, van Vollenhoven RF. Histopathologic and clinical outcome of rituximab treatment in patients with cyclophosphamide-resistant proliferative lupus nephritis. Arthritis Rheum. 2007;56(4):1263-1272. doi:10.1002/art.22505

39. Pepper R, Griffith M, Kirwan C, et al. Rituximab is an effective treatment for lupus nephritis and allows a reduction in maintenance steroids. Nephrol Dial Transplant. 2009;24(12):3717-3723. doi:10.1093/ndt/gfp336

40. Pinto LF, Velasquez CJ, Prieto C, Mestra L, Forero E, Marquez JD. Rituximab induces a rapid and sustained remission in Colombian patients with severe and refractory systemic lupus erythematosus. Lupus. 2011;20(11):1219-1226. doi:10.1177/0961203311409273

41. Ramos-Casals M, Soto MJ, Cuadrado MJ, Khamashta MA. Rituximab in systemic lupus erythematosus: a systematic review of off-label use in 188 cases. Lupus. 2009;18(9):767-776. doi:10.1177/ 0961203309106174

42. Roccatello D, Sciascia S, Rossi D, et al. Intensive short-term treatment with rituximab, cyclophosphamide and methylprednisolone pulses induces remission in severe cases of SLE with nephritis and avoids further immunosuppressive maintenance therapy. Nephrol Dial Transplant. 2011;26(12):3987-3992. doi:10.1093/ndt/gfr109

43. Terrier B, Amoura Z, Ravaud P, et al. Safety and efficacy of rituximab in systemic lupus erythematosus: results from 136 patients from the French autoimmunity and rituximab registry. Arthritis Rheum. 2010;62(8):2458-2466. doi:10.1002/art.27541

44. Trachana M, Koutsonikoli A, Farmaki E, Printza N, Tzimouli V, Papachristou F. Safety and efficacy of rituximab in refractory pediatric systemic lupus erythematosus nephritis: a single-center experience of Northern Greece. Rheumatol Int. 2013;33(3):809-813. doi:10.1007/s00296-011-2239-6

45. van Vollenhoven RF, Gunnarsson I, Welin-Henriksson E, et al. Biopsy-verified response of severe lupus nephritis to treatment with rituximab (anti-CD20 monoclonal antibody) plus cyclophosphamide after biopsy-documented failure to respond to cyclophosphamide alone. Scand J Rheumatol. 2004;33(6):423-427.

46. Merrill JT, Neuwelt CM, Wallace DJ, et al. Efficacy and safety of rituximab in moderately-to-severely active systemic lupus erythematosus: the randomized, double-blind, phase II/III systemic lupus erythematosus evaluation of rituximab trial. Arthritis Rheum. 2010;62(1):222-233. doi:10.1002/art.27233

47. Rovin BH, Furie R, Latinis K, et al. Efficacy and safety of rituximab in patients with active proliferative lupus nephritis: the Lupus nephritis assessment with rituximab study. Arthritis Rheum. 2012;64 (4):1215-1226. doi:10.1002/art.34359

48. Weidenbusch M, Rommele C, Schrottle A, Anders HJ. Beyond the LUNAR trial. Efficacy of rituximab in refractory lupus nephritis. Nephrol Dial Transplant. 2013;28(1):106-111. doi:10.1093/ndt/ gfs 285

49. Gomez Mendez LM, Cascino MD, Garg J, et al. Peripheral blood B cell depletion after rituximab and complete response in lupus nephritis. Clin J Am Soc Nephrol. 2018;13(10):1502-1509. doi:10.2215/CJN.01070118

50. Bekar KW, Owen T, Dunn R, et al. Prolonged effects of short-term anti-CD20 B cell depletion therapy in murine systemic lupus erythematosus. Arthritis Rheum. 2010;62(8):2443-2457. doi:10.1002/art.27515

51. Ahuja A, Shupe J, Dunn R, Kashgarian M, Kehry MR, Shlomchik MJ. Depletion of B cells in murine lupus: efficacy and resistance. $J$ Immunol. 2007;179(5):3351. doi:10.4049/ jimmunol.179.5.3351
52. Molloy ES, Calabrese LH. Progressive multifocal leukoencephalopathy associated with immunosuppressive therapy in rheumatic diseases: evolving role of biologic therapies. Arthritis Rheum. 2012;64 (9):3043-3051. doi:10.1002/art.34468

53. Faul C, Donnelly M, Merscher-Gomez S, et al. The actin cytoskeleton of kidney podocytes is a direct target of the antiproteinuric effect of cyclosporine A. Nat Med. 2008;14(9):931-938. doi:10.1038/nm.1857

54. Favre H, Miescher PA, Huang YP, Chatelanat F, Mihatsch MJ. Ciclosporin in the treatment of lupus nephritis. Am J Nephrol. 1989;9(Suppl 1):57-60. doi:10.1159/000168040

55. Dostal C, Tesar V, Rychlik I, et al. Effect of 1 year cyclosporine A treatment on the activity and renal involvement of systemic lupus erythematosus: a pilot study. Lupus. 1998;7(1):29-36. doi:10.1191/ 096120398678919714

56. Ogawa H, Kameda H, Nagasawa H, et al. Prospective study of low-dose cyclosporine $\mathrm{A}$ in patients with refractory lupus nephritis. Mod Rheumatol. 2007;17(2):92-97. doi:10.1007/s10165-006-0545-8

57. Liu Z, Zhang H, Liu Z, et al. Multitarget therapy for induction treatment of lupus nephritis: a randomized trial. Ann Intern Med. 2015;162(1):18-26. doi:10.7326/M14-1030

58. Lewis EJ, Hunsicker LG, Lan SP, Rohde RD, Lachin JM. A controlled trial of plasmapheresis therapy in severe lupus nephritis. The Lupus Nephritis Collaborative Study Group. $N$ Engl J Med. 1992;326(21):1373-1379. doi:10.1056/NEJM199205213262101

59. Braun NEC, Klein R, Kotter I, Saal J, Risler T. Immunoadsorption onto protein A induces remission in severe systemic lupus erythematosis. Nephrol Dial Transplant. 2000;15:1365-1372. doi:10.1093/ndt/15.9.1367

60. Soyuöz A, Ö K, Karaağaç T, Kılıç L, Ş A B, Özcebe O. Therapeutic plasma exchange for refractory SLE: a comparison of outcomes between different sub-phenotypes. Eur J Rheumatol. 2018;5 (1):32-36. doi:10.5152/eurjrheum.2017.17088

61. Kronbichler A, Brezina B, Quintana LF, Jayne DR. Efficacy of plasma exchange and immunoadsorption in systemic lupus erythematosus and antiphospholipid syndrome: a systematic review. Autoimmun Rev. 2016;15(1):38-49. doi:10.1016/j.autrev.2015.08.010

62. Dall'Era M, Chakravarty E, Wallace D, et al. Reduced B lymphocyte and immunoglobulin levels after atacicept treatment in patients with systemic lupus erythematosus: results of a multicenter, phase Ib, double-blind, placebo-controlled, dose-escalating trial. Arthritis Rheum. 2007;56(12):4142-4150. doi:10.1002/art.23047

63. Cheema GS, Roschke V, Hilbert DM, Stohl W. Elevated serum B lymphocyte stimulator levels in patients with systemic immune-based rheumatic diseases. Arthritis Rheum. 2001;44(6):1313-1319. doi:10.1002/1529-0131(200106)44:6<1313::AID-ART223>3.0.CO;2-S

64. Stohl W, Metyas S, Tan SM, et al. B lymphocyte stimulator overexpression in patients with systemic lupus erythematosus: longitudinal observations. Arthritis Rheum. 2003;48(12):3475-3486. doi:10.1002/art.11354

65. Furie R, Petri M, Zamani O, et al. A phase III, randomized, placebo-controlled study of belimumab, a monoclonal antibody that inhibits B lymphocyte stimulator, in patients with systemic lupus erythematosus. Arthritis Rheum. 2011;63(12):3918-3930. doi:10.1002/art.30613

66. Navarra SV, Guzman RM, Gallacher AE, et al. Efficacy and safety of belimumab in patients with active systemic lupus erythematosus: a randomised, placebo-controlled, phase 3 trial. Lancet. 2011;377 (9767):721-731. doi:10.1016/S0140-6736(10)61354-2

67. Dooley MA, Houssiau F, Aranow C, et al. Effect of belimumab treatment on renal outcomes: results from the phase 3 belimumab clinical trials in patients with SLE. Lupus. 2013;22(1):63-72. doi:10.1177/0961203312465781

68. ACCESS Trial Group. Treatment of lupus nephritis with abatacept: the abatacept and cyclophosphamide combination efficacy and safety study. Arthritis Rheumatol. 2014;66(11):3096-3104. doi:10.1002/ art.38790 
69. Jayne DDM, Wofsy D et al., editors. A phase III, randomized, double-blind, placebo-controlled study to evaluate the efficacy and safety of abatacept or placebo on standard of care in patients with active class III or IV lupus nephritis. ERA-EDTA Congress 2018; Copenhagen: Publication Number: LB03; 2018.

70. Wallace DJ, Kalunian K, Petri MA, et al. Efficacy and safety of epratuzumab in patients with moderate/severe active systemic lupus erythematosus: results from EMBLEM, a phase IIb, randomised, double-blind, placebo-controlled, multicentre study. Ann Rheum Dis. 2014;73(1):183-190. doi:10.1136/annrheumdis-2012-202760
71. Clowse ME, Wallace DJ, Furie RA, et al. Efficacy and safety of epratuzumab in moderately to severely active systemic lupus erythematosus: results from two phase III randomized, double-blind, placebo-controlled trials. Arthritis Rheumatol. 2017;69(2):362-375. doi:10.1002/art.39856

72. Merrill JT, Wallace DJ, Wax S, et al. Efficacy and safety of atacicept in patients with systemic lupus erythematosus: results of a twenty-four-week, multicenter, randomized, double-blind, placebo-controlled, parallel-arm, phase IIb study. Arthritis Rheumatol. 2018;70(2):266-276. doi:10.1002/art.40360

\section{Publish your work in this journal}

Open Access Rheumatology Research and Reviews is an international, peer-reviewed, open access journal publishing original research, reports, editorials, reviews and commentaries on all aspects of clinical and experimental rheumatology in the clinic and laboratory including the following topics: Pathology, pathophysiology of rheumatological diseases; Investigation, treatment and management of rheumatological diseases; Clinical trials and novel pharmacological approaches for the treatment of rheumatological disorders. The manuscript management system is completely online and includes a very quick and fair peer-review system, which is all easy to use. Visit http://www.dovepress.com/testimonials.php to read real quotes from published authors. 